\title{
Management of Animal Bite Wounds on Face: Our Experience
}

\author{
${ }^{1}$ Abhishek Mathur, ${ }^{2}$ Keerthi Ramesh, ${ }^{3}$ Anup Kumar G \\ ${ }^{1}$ Senior Resident, New Delhi Municipal Corporation (NDMS), New Delhi, India \\ ${ }^{2}$ Professor, Department of Oral and Maxillofacial Surgery, VS Dental College and Hospital, Bengaluru, Karnataka, India \\ ${ }^{3}$ Senior Lecturer, Department of Dentistry, Noorul Islam College of Dental Sciences (NICDS), Kerala, India
}

Correspondence: Abhishek Mathur, Senior Resident, New Delhi Municipal Corporation (NDMS), New Delhi, India e-mail: mathurabhishek82@gmail.com

\section{ABSTRACT}

Bite wounds are among the commonest types of trauma to which the man is subjected. Infection is the most common complication in animal bite wounds. The surgical treatment of facial animal bites remains a source of controversy. The controversies continue to center on the timing of wound debridement and primary wound closure as well as the use of antibiotic prophylaxis and primary plastic reconstruction. We report 30 cases of animal bites treated in our department and our protocol in the management of the same.

Keywords: Animal bites, Facial wounds.

\section{INTRODUCTION}

Bite wounds are among the commonest types of trauma to which the man is subjected. ${ }^{6}$ Biting is a natural instinct of animals and a large percentage of these bites are located on the face (15\%) thus, requiring intervention by an oral and maxillofacial surgeon. Dog bites account for 80 to $90 \%{ }^{1}$ of total bite cases and are mostly on lips, nose and cheeks. Children have more than twice the risk of adults and those aged 0 to 9 years are more likely to suffer higher injury rates. Bite wounds have always been considered complex injuries contaminated with a unique polymicrobial inoculums.

Animal bites can result in three main types of soft tissue trauma, namely punctures, lacerations and avulsions. Dog bites typically result in a combination of torn tissues and adjacent punctures, the so called 'hole-and-tear' effect. ${ }^{2}$ Crushing type of wounds may also be inflicted by dogs because of presence of rounded teeth and strong jaws. An adult dog can exert 200 pounds per square inch of pressure, with some large dogs able to exert $450 \mathrm{psi}^{4}{ }^{4}$ Such extreme pressure may damage deeper structures, such as bones, vessels, tendons, muscle and nerves.

The most common complication of animal bites is infection due to contamination of the wound by gram-positive and gramnegative microorganisms in the saliva. ${ }^{1}$ Because of high potential for infection, urgent attention is required with administration of antibiotics, thorough wound toilet and surgical debridement. Delayed repair is the traditional approach, especially for children who are presented late. Those patients reporting within 24 hours, primary repair of laceration is done after wound toilet, surgical debridement and postexposure rabies prophylaxis. This is the protocol in our unit and, we report here, experience with 30 cases seen in 3 years.

\section{PATIENT AND METHODS}

A total of 30 cases with facial wounds inflicted due to animal bites reported to the Emergency Department of Oral and
Maxillofacial Surgery, VS Dental College and Hospital, from June 2007 to June 2010.

Of the 30 cases reported, 20 were male and 10 were female with a range of 2 to 30 years and a mean age of 6 years (Fig. 1). A total of 27 patients reported within 24 hours after being bitten and three came with grossly infected wounds as late as 4 days after sustaining bites (Fig. 2). Cheek was the most common site involved ( $n=12$ ) with eight patients receiving multiple injuries on the face (Fig. 3). All the cases had injuries due to dog bite except in one patient in which horse was the culprit.

All the 30 patients had partial thickness injuries with none sustaining injuries to facial nerve, parotid duct or facial bones.

\section{OPERATIVE PROCEDURES}

All patients were treated under local anesthesia with primary closure of the wound except in three patients in which delayed

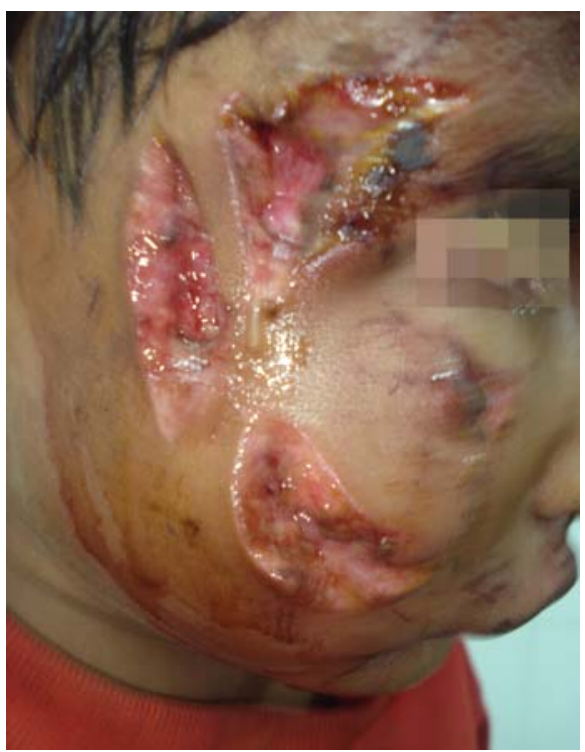

Fig. 1: Animal bite injury patient presenting immediately 


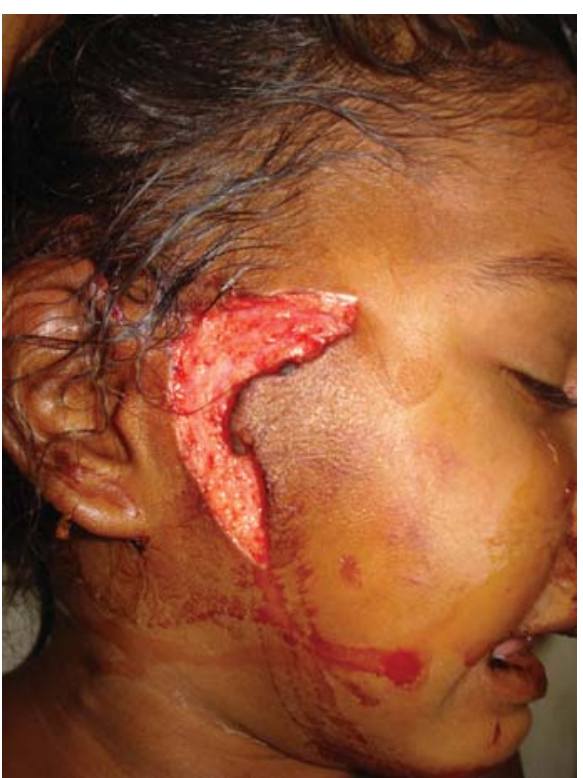

Fig. 2: Another young patient with dog bite injury

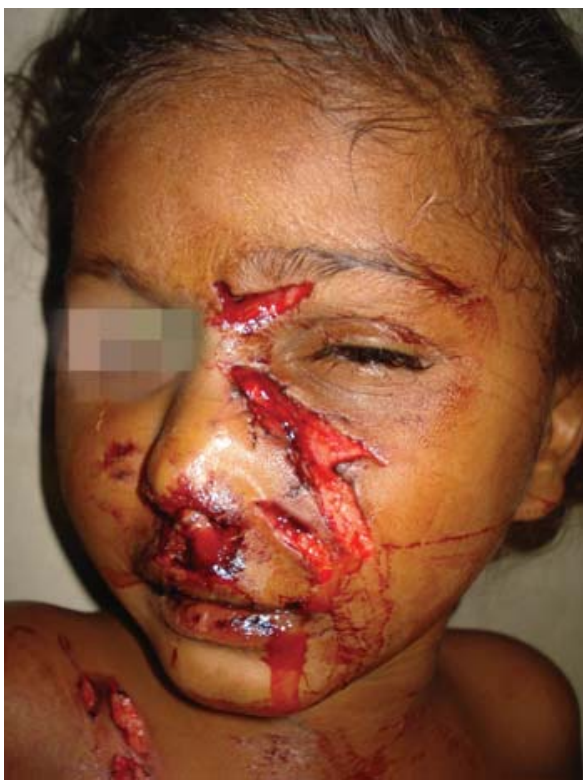

Fig. 3: Multiple facial bite injuries

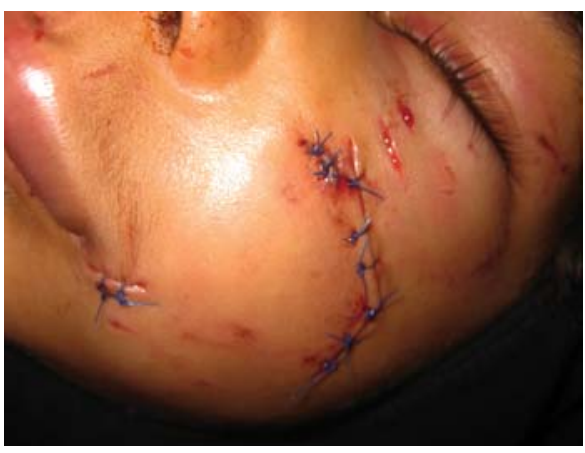

Fig. 4: Closure of the bite injuries

repair was done (Fig. 4). All patients initially had a thorough wound toilet with saline and surgical debridement of necrotic margins. Human rabies immune globulin(HRIG) was infiltrated into and around the wound as postexposure prophylaxis for rapid passive immune presentation. Dosage given of HRIG was
$20 \mathrm{IU} / \mathrm{kg}$ body weight. All the patients were then given postexposure rabies vaccination on $0,3,7$ and 14 days of exposure.

All the patients received oral antibiotic prophylaxis with augmentin plus metronidazole. In all the cases antibiotic prophylaxis was continued for 7 days.

\section{RESULTS}

Three of the 30 patients had infection with necrosis of wound margins in early postoperative phase which was treated with debridement and closure. In the follow-up period, one patient had hypertrophic scar which was treated with corticosteroid injections.

\section{DISCUSSION}

The surgical treatment of facial animal bites remains a source of controversy. The controversies continue to center on the timing of wound debridement and primary wound closure as well as the use of antibiotic prophylaxis and primary plastic reconstruction. The recommendations for wound closure vary from spontaneous healing to primary suturing with maximal tissue preservation. ${ }^{3}$ Different opinions can also be found in the literature regarding the prophylactic use of antibiotics.

It is important to avoid infection and to achieve an esthetically pleasing result in the case of animal bite patients. We recommend use of antibiotics in all our patients because none of the animal bite wounds or injuries can be considered either simple or clean. There has been up to 64 different species of bacteria isolated from the oral flora of dogs and several other human pathogens. ${ }^{4}$ Infections due to animal bites can be considered polymicrobial and no single species can be held accountable in the majority of the cases. ${ }^{5}$ The most commonly isolated aerobic bacteria from infected dog bites are alpha and beta hemolytic Streptococcus (24 to 46\%), Staphylococcus aureus and Staphylococcus epidermidis, Pasteurella multocida, E. coli, Pseudomonas and Moraxella species. The most common anaerobic bacteria isolated are Bacteroides, Prevotella and Fusobacterium species (13 to $76 \%) .{ }^{4}$ Augmentin and metronidazole ${ }^{7}$ are used in our department because of its activity against many of these and were given orally.

According to us, primary wound closure is the treatment of choice for all uninfected facial bite lacerations seen within 24 hours because this obtains the most favorable esthetic result. Subcutaneous sutures should be used sparingly. Deep puncture wounds should be left open. For patients presented to us after 'golden 24-hour period', we preferred delayed closure after 4 to 5 days. During this time, the wound was left open with moist dressings. In all the cases antirabies prophylaxis was given.

However, the most effective means to reduce wound infection in animal wound infection is by aggressive wound debridement at the time of surgical repair which is often neglected. Manual irrigation with a 19-gauge catheter on a 30 to $60 \mathrm{ml}$ syringe delivers a pressure range between 5 and 8 psi, considered optimal for appropriate decontamination. ${ }^{2}$ Normal 
saline is the fluid of choice for irrigation. ${ }^{2}$ Debridement of the facial wounds should be kept minimum so as to avoid sacrifice of tissue which has a good chance to survive.

\section{REFERENCES}

1. Javaid M, Feldberg L, Gipson M. Primary repair of dog bites to the face: 40 cases. J R Soc Med 1998;91:414-16.

2. Stefanopoulos Panagiotis K. Management of facial bite wounds. Dent Clin North America, Oct 2009;53(4):691-705.

3. Klaus-Dietrich Wolff. Management of animal bite injuries of the face: Experience with 94 patients. J Oral Maxillofac Surg 1998;56:838.
4. Morgan Jackson P, Haug Richard H, et al. Management of facial dog bite injuries. J Oral Maxillofac Surg 1995;53(4):435-41.

5. Ugoboko VI, Olasoji HO, Amole AOD. Facial injuries caused by animals in north Nigeria. Br J Oral Max Surg Oct 2002;40(5):433-37.

6. Stefanopoulos PK, Tarantzopoulou AD. Facial bite wounds: Management uptake. Int J Oral Maxillofac Surg 2005;34: 464-72.

7. Shubi Farrid M, Hamza Omar JM, Kalyanyama Boniphace M. Human bite injuries in the oro-facial region at the Muhimbili National Hospital, Tanzania. BMC Oral Health 2008;8:12. 